\title{
EIGENVALUES OF COUNTABLY CONDENSING MAPS
}

\author{
In-Sook Kim, Yun-Ho Kim, And Sunghui Kwon
}

\begin{abstract}
Using an index theory for countably condensing maps, we show the existence of eigenvalues for countably $k$-set contractive maps and countably condensing maps in an infinite dimensional Banach space $X$, under certain condition that depends on the quantitative characteristic, that is, the infimum of all $k \geq 1$ for which there is a countably $k$-setcontractive retraction of the closed unit ball of $X$ onto its boundary.
\end{abstract}

\section{Introduction}

A starting point of our investigation is a result of Guo [5] which states as follows:

Let $\Omega$ be a bounded open subset of an infinite dimensional Banach space $X$. If $F: \bar{\Omega} \rightarrow X$ is a compact map such that $\inf _{x \in \partial \Omega}\|F x\|>0$ and $F x \neq \lambda x$ for all $x \in \partial \Omega$ and all $\lambda \in(0,1]$, then ind $(F, \Omega)=0$.

It has been attempted to extend the result to strict-set contractions; see [2, $9,12]$. In a recent work [4], some generalizations to these contractions and condensing maps are obtained, under suitable condition that depends on the quantitative characteristic $R_{\gamma}$, where $\gamma$ is a measure of noncompactness on $X$. This means the infimum of all $k \geq 1$ for which there is a $k$ - $\gamma$-contractive retraction of the closed unit ball of $X$ onto its boundary. See [3, 4, 6, 11].

It is known in [5] that the above result is closely related to the problem of finding solutions for nonlinear equations. It is natural to consider this problem for a large class of countably condensing maps, roughly speaking, condensing on countable subsets of the space. The use of such countable sets to solve nonlinear equations can be found in [7].

Motivated by the work [4], our goal in the present paper is to study nonlinear eigenvalue problem for countably $k$-set contractive maps and countably condensing maps. To this end, we introduce an index theory for countably condensing maps due to Väth [10] and the corresponding characteristic $R_{\gamma}^{c}$ given in [6]. Using a result of [6], we give an extension of Guo's result to countably $k$-set contractive maps and obtain an eigenvalue result for these maps which includes the well known Birkhoff-Kellogg theorem as a special case. Moreover,

Received June 1, 2007; Revised September 12, 2007.

2000 Mathematics Subject Classification. 47J10, 47H09, 47H11.

Key words and phrases. eigenvalues, countably condensing maps, fixed point index. 
we present the existence of eigenvalues for countably condensing maps. As its application, we discuss nonlinear eigenvalue problem in a more concrete situation, as in $[4,5]$.

\section{An index theory}

In this section, we introduce a fixed point index theory for countably condensing maps due to Väth [10].

Given a nonempty subset $\Omega$ of a Banach space $X$, the closure, the boundary, the convex hull, and the closed convex hull of $\Omega$ in $X$ are denoted by $\bar{\Omega}, \partial \Omega$, $\operatorname{co} \Omega$, and $\overline{\operatorname{co}} \Omega$, respectively.

A function $\gamma:\{M \subset X: M$ is bounded $\} \rightarrow[0, \infty)$ is said to be a measure of noncompactness on $X$ if it satisfies the following properties:

(1) $\gamma(\overline{\mathrm{co}} M)=\gamma(M)$;

(2) $\gamma(M)=0$ if and only if $M$ is relatively compact;

(3) $\gamma(M \cup N)=\max \{\gamma(M), \gamma(N)\}$;

(4) $\gamma(M+N) \leq \gamma(M)+\gamma(N)$; and

(5) $\gamma(\alpha M)=|\alpha| \gamma(M)$ for all $\alpha \in \mathbb{R}$.

Note that the Kuratowski or the Hausdorff measure of noncompactness has the above properties; see [1].

Let $\Omega$ be a nonempty subset of $X$ and $\gamma$ a measure of noncompactness on $X$, and $k \geq 0$. A continuous map $F: \Omega \rightarrow X$ is said to be countably $k$ - $\gamma$-contractive if $\gamma(F(C)) \leq k \gamma(C)$ for each countable bounded set $C \subset \Omega$. A continuous map $F: \Omega \rightarrow X$ is said to be countably $\gamma$-condensing if $\gamma(F(C))<\gamma(C)$ for each countable bounded set $C \subset \Omega$ with $\gamma(C)>0$. Moreover, if $\gamma(F(B)) \leq k \gamma(B)$ for all bounded sets $B \subset \Omega$, then $F$ is called $k$ - $\gamma$-contractive. If $\gamma(F(B))<\gamma(B)$ for all bounded sets $B \subset \Omega$ with $\gamma(B)>0$, then $F$ is called $\gamma$-condensing.

A continuous homotopy $H:[0,1] \times \Omega \rightarrow X$ is said to be countably $\gamma$ condensing if $\gamma(H([0,1] \times C))<\gamma(C)$ for each countable bounded set $C \subset \Omega$ with $\gamma(C)>0$.

To define an index for countably condensing maps, we need the following result which is a particular form of Corollary 2.1 in [10].

Proposition 2.1. Let $\Omega$ be an open bounded nonempty subset of a Banach space $X$. If $F: \bar{\Omega} \rightarrow X$ is a countably $\gamma$-condensing map, then there exists a compact convex nonempty subset $S$ of $X$ such that

(1) $F(\bar{\Omega} \cap S)$ is a subset of $S$; and

(2) the relation $x \in \overline{\mathrm{co}}[\{F x\} \cup S]$ implies $x \in S$.

Such a closed convex nonempty set $S$ is called a fundamental set for $F$.

Let $\Omega$ be an open bounded nonempty set in a Banach space $X$. Suppose that $F: \bar{\Omega} \rightarrow X$ is a countably $\gamma$-condensing map such that $F x \neq x$ for all 
$x \in \partial \Omega$. In view of Proposition 2.1, there exists a compact fundamental set $S$ for $F$ such that

$$
F(\bar{\Omega} \cap S) \subset S .
$$

Let $R: X \rightarrow S$ be any retraction onto $S$. Since $I-F \circ R \neq 0$ on the boundary of $R^{-1}(\Omega)$, the Leray-Schauder degree for the compact map $F \circ R$, $d_{L S}\left(I-F \circ R, R^{-1}(\Omega), 0\right)$, is defined. Now we define a fixed point index for the countably $\gamma$-condensing map $F$ as

$$
\text { ind }(F, \Omega):=d_{L S}\left(I-F \circ R, R^{-1}(\Omega), 0\right) \text {. }
$$

The fixed point index for $\gamma$-condensing maps, where $\gamma$ is the Kuratowski measure of noncompactness, has been developed by Nussbaum [8]. In case when $F$ is a compact map, the above indices agree with the Leray-Schauder degree.

We give some properties of the above index which will be used later; see $[10$, Theorem 1.3].

Proposition 2.2. Let $\Omega$ be an open bounded nonempty set in a Banach space $X$. Let $F: \bar{\Omega} \rightarrow X$ be a countably $\gamma$-condensing map such that $F x \neq x$ for all $x \in \partial \Omega$. Then it has the following properties:

(1) If ind $(F, \Omega) \neq 0$, then $F$ has a fixed point in $\Omega$.

(2) (Normalization) If $F \equiv 0$ and $0 \in \Omega$, then $\operatorname{ind}(F, \Omega)=1$.

(3) (Homotopy invariance) If $H:[0,1] \times \bar{\Omega} \rightarrow X$ is a countably $\gamma$-condensing homotopy such that $H(t, x) \neq x$ for all $(t, x) \in[0,1] \times \partial \Omega$, then

$$
\text { ind }(H(0, \cdot), \Omega)=\text { ind }(H(1, \cdot), \Omega) \text {. }
$$

(4) If $G: \bar{\Omega} \rightarrow X$ is a countably $\gamma$-condensing map such that $G x=F x$ for all $x \in \partial \Omega$, then ind $(G, \Omega)=$ ind $(F, \Omega)$.

Let $X$ be an infinite dimensional Banach space. We denote the closed ball of radius $r>0$ by $B_{r}(X)=\{x \in X:\|x\| \leq r\}$ and its boundary by $S_{r}(X)$, respectively.

Consider the following two quantitative characteristics:

$$
\begin{aligned}
R_{\gamma}(X)=\inf \{k \geq 1: & \text { there is a } k \text { - } \gamma \text {-contractive retraction } \\
& \left.R: B_{1}(X) \rightarrow S_{1}(X)\right\} \\
R_{\gamma}^{c}(X)=\inf \{k \geq 1: & \text { there is a countably } k \text { - } \gamma \text {-contractive } \\
& \text { retraction } \left.R: B_{1}(X) \rightarrow S_{1}(X)\right\}
\end{aligned}
$$

and $R_{\gamma}^{c}(X)=\infty$ if no such a contractive retraction exists. The fact that $k \geq 1$ is always possible, by Mönch's fixed point theorem [7]; see also [10]. It is obvious that $R_{\gamma}(X) \geq R_{\gamma}^{c}(X) \geq 1$. For example, if $\gamma$ is the Hausdorff measure of noncompactness, then $R_{\gamma}^{c}(C([0,1]))=R_{\gamma}(C([0,1]))=1$ and $R_{\gamma}^{c}\left(L_{p}([0,1])\right)=$ $R_{\gamma}\left(L_{p}([0,1])\right)=1(1 \leq p<\infty)$; see $[3,11]$.

In what follows, $\Omega$ will always be a bounded open subset of an infinite dimensional Banach space $X$ which contains the origin 0. For simplicity, we write $R_{\gamma}^{c}$ for $R_{\gamma}^{c}(X)$. 


\section{The countably $k$-set contractive case}

In this section, we apply index theory to show the existence of an eigenvalue for countably $k$-set contractive maps, where the condition depends on the characteristic $R_{\gamma}^{c}$.

The following lemma stated in [6, Theorem 5] is a key tool in proving our results. For completeness we give the proof here; see also [4, Lemma 3.1].

Lemma 3.1. Let $F: \bar{\Omega} \rightarrow X$ be a countably $k$ - $\gamma$-contractive map for some $k>0$ such that $k R_{\gamma}^{c}<1$. Suppose that

$$
\inf _{x \in \partial \Omega}\|F x\|>\|y\| \quad \text { for all } y \in \Omega
$$

and $F$ has no fixed points on $\partial \Omega$. Then ind $(F, \Omega)=0$.

Proof. Choose a real number $k_{1}$ such that $k_{1} \geq R_{\gamma}^{c}, k k_{1}<1$, and there is a countably $k_{1}-\gamma$-contractive retraction $R: B_{1}(X) \rightarrow S_{1}(X)$. Set $\delta:=$ $\inf _{x \in \partial \Omega}\|F x\|$ and define $R_{\delta}: B_{\delta}(X) \rightarrow S_{\delta}(X)$ by

$$
R_{\delta}(x):=\delta R\left(\frac{x}{\delta}\right) \quad \text { for } x \in B_{\delta}(X) .
$$

It is easily checked that $R_{\delta}$ is also a countably $k_{1}-\gamma$-contractive retraction. Consider a map $G: \bar{\Omega} \rightarrow X$ defined by

$$
G x:=L(F x) \quad \text { for } x \in \bar{\Omega},
$$

where

$$
L x= \begin{cases}R_{\delta}(x) & \text { if } x \in B_{\delta}(X), \\ x & \text { if } x \in X \backslash B_{\delta}(X) .\end{cases}
$$

Then $G$ is countably $k k_{1}-\gamma$-contractive and hence countably $\gamma$-condensing and we have

$$
\inf _{x \in \bar{\Omega}}\|G x\| \geq \delta>\|y\| \quad \text { for all } y \in \Omega .
$$

Since $G=F$ on $\partial \Omega$ and $G$ has no fixed points in $\Omega$, it follows from Proposition 2.2 that

$$
\text { ind }(F, \Omega)=\operatorname{ind}(G, \Omega)=0 \text {. }
$$

We first give an extension of Guo's result to countably $k$ - $\gamma$-contractive maps which is analogous to Theorem 3.2 of [4] when $F$ is a $k-\gamma$-contraction.

Theorem 3.2. Suppose that $F: \bar{\Omega} \rightarrow X$ is a countably $k$ - $\gamma$-contractive map for some $k>0$ such that $k R_{\gamma}^{c}<1$ and it satisfies

$$
\inf _{x \in \partial \Omega}\|F x\|>k R_{\gamma}^{c} \sup _{y \in \Omega}\|y\| .
$$

If $F x \neq \lambda x$ for all $x \in \partial \Omega$ and all $\lambda \in\left(k R_{\gamma}^{c}, 1\right]$, then ind $(F, \Omega)=0$. 
Proof. We can find a real number $\varepsilon>0$ such that $k R_{\gamma}^{c}+\varepsilon<1$ and

$$
\inf _{x \in \partial \Omega}\|F x\|>\left(k R_{\gamma}^{c}+\varepsilon\right) \sup _{y \in \Omega}\|y\| .
$$

Set $k_{1}:=k /\left(k R_{\gamma}^{c}+\varepsilon\right)$ and define a map $G: \bar{\Omega} \rightarrow X$ by

$$
G x:=\frac{1}{k R_{\gamma}^{c}+\varepsilon} F x \quad \text { for } x \in \bar{\Omega} .
$$

Then $G$ is countably $k_{1}-\gamma$-contractive, $k_{1} R_{\gamma}^{c}<1$, and $G$ has no fixed points on $\partial \Omega$, by taking $\lambda=k R_{\gamma}^{c}+\varepsilon$ in the hypothesis. Lemma 3.1 implies that ind $(G, \Omega)=0$. Consider a homotopy $H:[0,1] \times \bar{\Omega} \rightarrow X$ defined by

$$
H(t, x):=t F x+(1-t) G x \quad \text { for }(t, x) \in[0,1] \times \bar{\Omega} .
$$

Then $H$ is countably $\gamma$-condensing. In fact, for each countable set $C \subset \bar{\Omega}$ with $\gamma(C)>0$ we have

$$
\begin{aligned}
\gamma(H([0,1] \times C)) & \leq \gamma(\operatorname{co}[F(C) \cup G(C)]) \\
& \leq \max \{\gamma(F(C)), \gamma(G(C))\} \\
& <\gamma(C)
\end{aligned}
$$

because $F$ and $G$ are countably $\gamma$-condensing on $\bar{\Omega}$. As in the proof of Theorem 3.2 in [4], we have by hypothesis

$$
F x \neq\left(t+\frac{1-t}{k R_{\gamma}^{c}+\varepsilon}\right)^{-1} x, \quad \text { that is, } \quad H(t, x) \neq x
$$

for all $(t, x) \in[0,1] \times \partial \Omega$. We conclude by Proposition 2.2 that

$$
\text { ind }(F, \Omega)=\operatorname{ind}(G, \Omega)=0 \text {. }
$$

The following statement is Lemma 1 of [5] given in the introduction.

Corollary 3.3. Suppose that $F: \bar{\Omega} \rightarrow X$ is a compact map that satisfies

$$
\inf _{x \in \partial \Omega}\|F x\|>0 \text {. }
$$

If $F x \neq \lambda x$ for all $x \in \partial \Omega$ and all $\lambda \in(0,1]$, then ind $(F, \Omega)=0$.

Proof. Choose a real number $k>0$ such that $k R_{\gamma}^{c}<1$ and

$$
\inf _{x \in \partial \Omega}\|F x\|>k R_{\gamma}^{c} \sup _{y \in \Omega}\|y\| .
$$

Theorem 3.2 is applicable because $F$ is countably $k$ - $\gamma$-contractive.

Now we show the existence of an eigenvalue for countably $k$ - $\gamma$-contractive maps; see [4, Corollary 3.5] for $k$ - $\gamma$-contractions.

Theorem 3.4. Let $F: \bar{\Omega} \rightarrow X$ be a countably $k$ - $\gamma$-contractive map for any $k>0$. If

$$
\inf _{x \in \partial \Omega}\|F x\|>k R_{\gamma}^{c} \sup _{y \in \Omega}\|y\|,
$$

then there exist a real number $\lambda>k R_{\gamma}^{c}$ and a vector $x \in \partial \Omega$ such that $F x=\lambda x$. 
Proof. Assume on the contrary that

$$
F x \neq \lambda x \quad \text { for all } x \in \partial \Omega \text { and all } \lambda>k R_{\gamma}^{c} .
$$

Choose a real number $\varepsilon>0$ such that

$$
\inf _{x \in \partial \Omega}\|F x\|>\left(k R_{\gamma}^{c}+\varepsilon\right) \sup _{y \in \Omega}\|y\| .
$$

Let $G: \bar{\Omega} \rightarrow X$ be a countably $\gamma$-condensing map defined by

$$
G x:=\frac{1}{k R_{\gamma}^{c}+\varepsilon} F x \quad \text { for } x \in \bar{\Omega} .
$$

As in the proof of Theorem 3.2, a similar argument shows that ind $(G, \Omega)=0$. Now consider a countably $\gamma$-condensing homotopy $H:[0,1] \times \bar{\Omega} \rightarrow X$ defined by

$$
H(t, x):=(1-t) G x \quad \text { for }(t, x) \in[0,1] \times \bar{\Omega} .
$$

Our assumption implies that $H(t, x) \neq x$ for all $x \in \partial \Omega$ and $t \in[0,1]$. From $H(1, x)=0$ for all $x \in \bar{\Omega}$ and $0 \in \Omega$, it follows by Proposition 2.2 that

$$
\text { ind }(G, \Omega)=\operatorname{ind}(H(1, \cdot), \Omega)=1 \text {, }
$$

which is impossible. We conclude that there exist a real number $\lambda>k R_{\gamma}^{c}$ and a point $x \in \partial \Omega$ such that $F x=\lambda x$.

Our result reduces to the well known Birkhoff-Kellogg theorem.

Corollary 3.5. If $F: \bar{\Omega} \rightarrow X$ is a compact map such that

$$
\inf _{x \in \partial \Omega}\|F x\|>0 \text {, }
$$

then $F$ has at least an eigenvalue $\lambda>0$ whose corresponding vector $x$ lies in $\partial \Omega$.

Proof. Choose a real number $k>0$ such that

$$
\inf _{x \in \partial \Omega}\|F x\|>k R_{\gamma}^{c} \sup _{y \in \Omega}\|y\| .
$$

Apply Theorem 3.4.

\section{The countably condensing case}

In this section, we present an eigenvalue result for countably condensing maps. As its application, we study nonlinear eigenvalue problem in a more concrete situation.

We give a simple proof of the following countably condensing version; compare with Theorem 4.1 of [4] in the condensing case.

Theorem 4.1. Suppose that $F: \bar{\Omega} \rightarrow X$ is a countably $\gamma$-condensing map that satisfies

$$
\inf _{x \in \partial \Omega}\|F x\|>R_{\gamma}^{c} \sup _{y \in \Omega}\|y\|
$$

Then ind $(F, \Omega)=0$. 
Proof. Choose $\varepsilon>0$ such that

$$
\inf _{x \in \partial \Omega}\|F x\|>\left(R_{\gamma}^{c}+\varepsilon\right) \sup _{y \in \Omega}\|y\| .
$$

Set $k:=1 /\left(R_{\gamma}^{c}+\varepsilon\right)$ and define $G: \bar{\Omega} \rightarrow X$ by

$$
G x:=k F x \quad \text { for } x \in \bar{\Omega} .
$$

Then $G$ is countably $k$ - $\gamma$-contractive. It is easily verified that

$$
\inf _{x \in \partial \Omega}\|G x\|>\sup _{x \in \partial \Omega}\|x\| .
$$

Since $k R_{\gamma}^{c}<1$ and $G$ has no fixed points on $\partial \Omega$, Lemma 3.1 implies that ind $(G, \Omega)=0$. Consider a homotopy $H:[0,1] \times \bar{\Omega} \rightarrow X$ defined by

$$
H(t, x):=t F x+(1-t) G x \quad \text { for }(t, x) \in[0,1] \times \bar{\Omega} \text {. }
$$

As before, $H$ is obviously countably $\gamma$-condensing. For all $(t, x) \in[0,1] \times \partial \Omega$, we have

$$
\|H(t, x)\|=\|(t+k(1-t)) F x\| \geq k\|F x\|=\|G x\|>\|x\|
$$

and thus $H(t, x) \neq x$. Consequently, Proposition 2.2 implies that

$$
\text { ind }(F, \Omega)=\text { ind }(G, \Omega)=0 \text {. }
$$

Now we have an existence result on eigenvalues of countably condensing maps; see [4, Corollary 4.2] for condensing maps.

Theorem 4.2. Let $F: \bar{\Omega} \rightarrow X$ be a countably $\gamma$-condensing map such that

$$
\inf _{x \in \partial \Omega}\|F x\|>R_{\gamma}^{c} \sup _{y \in \Omega}\|y\| .
$$

Then there exist a real number $\lambda>R_{\gamma}^{c}$ and a vector $x \in \partial \Omega$ such that $F x=\lambda x$.

Proof. Notice that each countably $\gamma$-condensing map is countably 1 - $\gamma$-contractive. Applying Theorem 3.4, the conclusion follows.

As an application of Theorem 4.2, we observe nonlinear eigenvalue problem in a concrete situation; see $[4,5]$.

Theorem 4.3. Let $X=L_{p}([0,1])(p>1)$ and let $\Omega$ be a bounded open subset of $X$ with $0 \in \Omega$. Assume that

(1) $k:[0,1] \times[0,1] \rightarrow \mathbb{R}$ is a continuous nonnegative function such that

$$
\alpha=\min \left\{\int_{0}^{1} k(t, s) d t: s \in[0,1]\right\}>0 .
$$

(2) $L: \bar{\Omega} \rightarrow X$ is an integral operator defined by

$$
L x(t):=\int_{0}^{1} k(t, s)|x(s)|^{p} d s \quad \text { for } t \in[0,1] .
$$

(3) $G: \bar{\Omega} \rightarrow X$ is a countably $\gamma$-condensing map such that $G(\partial \Omega)$ is bounded, where $\gamma$ is the Hausdorff measure of noncompactness. 
Consider nonlinear operator $F: \bar{\Omega} \rightarrow X$ given by

$$
F x=L x+G x .
$$

Set

$$
\beta=\inf _{x \in \partial \Omega}\|x\|, \quad d=\sup _{y \in \Omega}\|y\|, \quad \text { and } \delta=\sup _{x \in \partial \Omega}\|G x\| .
$$

If $\alpha \beta^{p}>\delta+d$, then $F$ has a positive eigenvalue whose corresponding vector lies in $\partial \Omega$.

Proof. For each $x \in \bar{\Omega}$, we obtain from Jensen's inequality that

$$
\begin{aligned}
\alpha\|x\|^{p} & =\int_{0}^{1} \alpha|x(s)|^{p} d s \leq \int_{0}^{1}\left(\int_{0}^{1} k(t, s) d t\right)|x(s)|^{p} d s \\
& =\int_{0}^{1} \int_{0}^{1} k(t, s)|x(s)|^{p} d s d t \\
& \leq\left(\left.\left.\int_{0}^{1}\left|\int_{0}^{1} k(t, s)\right| x(s)\right|^{p} d s\right|^{p} d t\right)^{\frac{1}{p}}=\|L x\| .
\end{aligned}
$$

For all $x \in \partial \Omega$, we have

$$
\|F x\| \geq\|L x\|-\|G x\| \geq \alpha \beta^{p}-\delta>d
$$

and hence

$$
\inf _{x \in \partial \Omega}\|F x\|>\sup _{y \in \Omega}\|y\| .
$$

Notice that $R_{\gamma}^{c}\left(L_{p}([0,1])\right)=R_{\gamma}\left(L_{p}([0,1])\right)=1$ by Theorem 4.5 of [3]. Since $L$ is compact and hence $F$ is countably $\gamma$-condensing, Theorem 4.2 implies that $F$ has an eigenvalue $\lambda>0$ with corresponding vector $x \in \partial \Omega$.

In addition, a similar argument shows that Theorem 4.3 holds provided that $G$ is a countably $k$ - $\gamma$-contractive map and $\alpha \beta^{p}>\delta+k d$, where Theorem 3.4 is used in place of Theorem 4.2.

Acknowledgment. This work is supported by the BK 21 Mathematical Modelling HRD Division, Sungkyunkwan University.

\section{References}

[1] J. M. Ayerbe Toledano, T. Domínguez Benavides, and G. López Acedo, Measures of Noncompactness in Metric Fixed Point Theory, Operator Theory: Advances and Applications, 99. Birkhäuser Verlag, Basel, 1997.

[2] F. E. Browder, Remarks on the paper: "Boundary conditions for condensing mappings" [Nonlinear Anal. 8 (1984), no. 3, 209-219] by Q. Y. Yu and Browder, Nonlinear Anal. 8 (1984), no. 9, 1113.

[3] D. Caponetti, A. Trombetta, and G. Trombetta, Proper 1-ball contractive retractions in Banach spaces of measurable functions, Bull. Austral. Math. Soc. 72 (2005), no. 2, 299-315.

[4] - An extension of Guo's theorem via $k-\psi$-contractive retractions, Nonlinear Anal. 64 (2006), no. 9, 1897-1907. 
[5] D. Guo, Eigenvalues and eigenvectors of nonlinear operators, Chin. Ann. Math. 2 (1981), 65-80.

[6] I.-S. Kim and M. Väth, Some remarks on measures of noncompactness and retractions onto spheres, Topology Appl. 154 (2007), no. 17, 3056-3069.

[7] H. Mönch, Boundary value problems for nonlinear ordinary differential equations of second order in Banach spaces, Nonlinear Anal. 4 (1980), no. 5, 985-999.

[8] R. D. Nussbaum, The fixed point index for local condensing maps, Ann. Mat. Pura Appl. (4) 89 (1971), 217-258.

[9] Y. Sun, A generalization of Guo's theorem and applications, J. Math. Anal. Appl. 126 (1987), no. 2, 566-573.

[10] M. Väth, Fixed point theorems and fixed point index for countably condensing maps, Topol. Methods Nonlinear Anal. 13 (1999), no. 2, 341-363.

[11] J. Wośko, An example related to the retraction problem, Ann. Univ. Mariae CurieSkłodowska Sect. A 45 (1991), 127-130.

[12] Q. Y. Yu and F. E. Browder, Boundary conditions for condensing mappings, Nonlinear Anal. 8 (1984), no. 3, 209-219.

IN-SOOK KIM

Department of Mathematics

SUNGKYUNKWAN UNIVERSITY

SuWON 440-746, KorEA

E-mail address: iskim@skku.edu

Yun-Ho KIM

Department of Mathematics

SUNGKYUNKWAN UNIVERSITY

SuWON 440-746, KoreA

E-mail address: kyh1213@skku.edu

Sunghui KWON

Department of Mathematics

SUNGKYUNKWAN UNIVERSITY

SuWON 440-746, Korea

E-mail address: shkwon99@skku.edu 